\title{
Research and Practice of the Integration of Theory and Practice Teaching Method Based on the Life Cycle of Medical Device Products: A Case Study of Medical Optical Instrument
}

Shijun Guo ${ }^{1, \mathrm{a}, *}$, Geer Yang ${ }^{1, \mathrm{~b}}$, Jie Lyu ${ }^{1, \mathrm{c}}$, Hao Ding ${ }^{1, \mathrm{~d}}$, Anmin Peng ${ }^{1, \mathrm{e}}$, Yang Liu ${ }^{1, \mathrm{f}}$ and Dan Lyu ${ }^{1, g}$

\begin{abstract}
${ }^{1}$ School of Medical Instrumentation, Shanghai University of Medicine \& Health Sciences, Shanghai, P.R.China aguosj@sumhs.edu.cn, ${ }^{b} y a n g g e @ s u m h s . e d u . c n,{ }^{c} l v j @ s u m h s . e d u . c n,{ }^{d} d i n g h @ s u m h s . e d u . c n,{ }^{e}$ pengam@sumhs.edu.cn, liuy@sumhs.edu.cn, ${ }^{g} l v d @$ sumhs.edu.cn

*Corresponding author
\end{abstract}

\begin{abstract}
Based on the core purpose of teaching, this paper reviews the relationship between teaching methods and teaching tasks. From the perspective of educational technology, combining the background of precision medical device technology specialty, industry characteristics and students' future development characteristics, this paper explores an integration of theory and practice teaching method based on the life cycle of medical device products. The course development of medical optical instrument is introduced in detail from two levels of teaching overall design and teaching unit design. In this paper, research and practice of the integration of theory and practice teaching method based on the life cycle of medical device products, could provide a new way of thinking for the technology applied talents cultivation in the field of medical device, including medical optical instrument, and other products, through each link such as research and development, installation and debugging, quality inspection, maintenance, clinical management and marketing service.
\end{abstract}

Keywords: medical device, life cycle, integration of theory and practice teaching method, medical optical instrument

\section{基于医疗器械产品生命周期的理实融合教学方法研究与 实践一一以《医用光学仪器》为例 郭世俊 $^{1, \mathrm{a},{ }^{*}}$ ，杨戈尔 ${ }^{1, \mathrm{~b}}$ ，吕杰 ${ }^{1, \mathrm{c}}$ ，丁皓 ${ }^{1, \mathrm{~d}}$, 彭安民 ${ }^{1, \mathrm{e}}$, 刘杨 ${ }^{1, \mathrm{f}}$ ，吕丹 ${ }^{1, \mathrm{~g}}$}

\footnotetext{
${ }^{1}$ 医疗器械学院, 上海健康医学院, 上海, 中国

${ }^{a}$ guosj@sumhs.edu.cn, ${ }^{b} y a n g g e @ s u m h s . e d u . c n,{ }^{c} l v j @ s u m h s . e d u . c n,{ }^{d} d i n g h @ s u m h s . e d u . c n,{ }^{e}$ pengam@sumhs.edu.cn, fliuy@sumhs.edu.cn, ${ }^{g} l v d @$ sumhs.edu.cn

*通讯作者
}

\section{摘要}

本文从教学的核心目的出发, 重新审视了教学方法与教学任务的关系。并从教育技术学的角度, 结合 精密医疗器械技术专业背景、行业特色与学生未来发展特点, 探索了一种基于医疗器械产品生命周期 的理实融合教学方法。通过教学实践举例, 从教学整体设计和教学单元设计两个层次详细介绍了医用 光学仪器课程开发情况。本文提出的基于医疗器械产品生命周期的理实融合教学方法的研究与实践, 可为培养包括医用光学仪器等产品在内的医疗器械领域的研发生产、安装调试、质量检测、技术维护、 临床管理和营销服务各环节的高素质技术应用型人才提供一种新思路。

关键词：医疗器械，生命周期，理实融合教学方法，医用光学仪器 


\subsection{3. 任务完成的多样开放性}

\section{1. 引言}

教学的核心目的是培养学生的概念理解力, 认知技能和方 法, 而非获取事实性的现成信息 [1]。而掌握新知识又是 学生学习的目标之一, 如何让学生在课堂教学中更高效的 学习, 从而实现知识的传授、能力的培养、素质的提升是 教学研究与课程建设的初心和使命。本文在学习现代高等 教育先进教学理念的基础上, 结合专业背景、行业特色与 学生未来发展特点, 总结课程教学经验, 在《医用光学仪 器》课程教学中探索了基于医疗器械产品生命周期的理实 融合教学方法。

\section{2. 基于医疗器械产品生命周期的理实融合教学 方法}

\section{1. 医疗器械产品生命周期}

我国《医疗器械监督管理条例》等法规体系中, 对医疗器 械的研制、分类、临床试验、注册、生产、经营、使用、 不良事件监测和再评价以及召回等环节作了针对性的规 定, 反映了医疗器械全生命周期管理的要求[2]。2019 年 国家药品监督管理局强调, 在监管工作中引入更科学、更 新颖的方法, 以科学的态度、专业的精神, 加强医疗器械 全生命周期的风险防控, 提高医疗器械质量安全水平, 满 足临床使用需求。这些都对现代医疗器械人才的培养提出 了新的知识、能力和素质要求。

\section{2. 理实融合教学方法反思}

理实融合教学方法能够将知识概念、认知技能和方法充分 融合在一起, 通过教学活动达成 “知识、能力、素质” 三 位一体式的培养目标[3]。在教学实践中, 教学方法、教 学策略是形式要素, 而教学任务、问题是内容要素 [4]。 从能力培养角度, 学生在教育过程中的自觉思考是关键。 因此, 教学活动中要提供好的任务和问题, 一般具有下列 三个基本特征。

\subsection{1. 知识运用的真实对应性}

教学实践中工作任务的完成和相应问题的解决是真实运 用相关特定知识的结果, 而非仅仅依赖经验猜测或基于生 活常识。

\subsection{2. 情境信息的取实丰富性}

教学实践中学习和工作情境的选取来源于实在的案例, 与 现实场景有很强的联系性, 可信并易于知识同化, 即把新 知识向原有框架里装。
教学实践中工作任务的完成和相应问题的解决有着多条 可以选择的路径, 在设计相应任务时应充分考虑到思维的 开放性。

\section{3. 教学实践举例一一医用光学仪器}

\section{1. 医用光学仪器课程定位}

\subsection{1. 精密医疗器械技术专业教学要求}

上海健康医学院精密医疗器械技术专业教学计划指出 [5], 学生应具备生物学、医学、光机电工程技术有关的 较为宽广的理论知识, 具有创新能力和实践能力, 德智体 美全面发展, 具备医疗器械产品制造和维修的基础知识与 基本技能, 能从事以手术及急救设备、临床检验分析设备、 医用光学仪器等产品为主的局部设计与制造、安装调试、 质量检测、技术维护、临床管理和营销服务一线的高素质 技术应用型人才。

\subsection{2. 医用光学仪器课程任务}

医用光学仪器广泛应用于生理及医学研究; 检验科、病理 科等医技科室; 消化科、泌尿科、眼科、耳鼻喉科等临床 科室。《医用光学仪器》是精密医疗器械技术 (精密医疗 器械方向) 专业的一门专业课程。本课程主要内容包括理 论部分: 几何光学、物理光学的基本原理, 光学材料与光 学元器件, 和仪器部分: 生物和医用显微镜、医用内窥镜 和眼科光学仪器的应用及维护。通过本课程的学习, 使学 生具备操作、保养和维护医用光学仪器的基本技能和方 法, 为学生在医用光学仪器领域工作打下基础[6]。

\section{2. 医用光学仪器教学设计}

\subsection{1. 教学整体设计}

根据 “以就业为导向、以能力为本位、以发展为核心” 的 基于工作过程的课程单元设计思路 [7], 本课程教学内容 上可以归纳为 “两基三仪” , 即两大基本原理: 几何光学 基本原理、物理光学基本原理, 以及三种典型仪器: 生物 和医用显微镜、医用内窥镜、眼科光学仪器。通过进一步 分析设计, 引入理实融合项目化教学, 实行教学做一体教 学, 由 5 个理实融合模块组成, 每个理实融合模块包含 2 个理实融合项目, 见表 1 。 
表 1 教学整体设计一一理实融合模块及项目列表

\begin{tabular}{|l|l|l|}
\hline \multicolumn{1}{|c|}{ 序号 } & \multicolumn{1}{|c|}{ 理实融合模块 } & \multicolumn{1}{c|}{ 理实融合项目 } \\
\hline 模块一 & 光学材料及零件认知 & $\begin{array}{l}\text { 项目 1: 光学材料、光学零件的识别和清洁 } \\
\text { 项目 2: 光学透镜组的分解与组装 }\end{array}$ \\
\hline 模块二 & 生物医学光学图像获取及设备维护 & $\begin{array}{l}\text { 项目 3: 倒置型生物显微镜的认识与操作 } \\
\text { 项目 4: 生物显微镜的拆装 }\end{array}$ \\
\hline 模块三 & 医用内窥镜操作及设备维护 & $\begin{array}{l}\text { 项目 5: 硬性内窥镜 }(\text { 膀胱镜 }) \text { 的操作与维护 } \\
\text { 项目 } 6: \text { 电子内窥镜 (胃镜) 的操作与维护 }\end{array}$ \\
\hline 模块四 & 眼科检查与测量及设备维护 & $\begin{array}{l}\text { 项目 7: 检眼镜的操作与维护 } \\
\text { 项目 8: 裂隙灯显微镜的使用 }\end{array}$ \\
\hline 模块五 & 眼科验光及设备维护 & $\begin{array}{l}\text { 项目 9: 检影镜模拟验光 } \\
\text { 项目 10: 综合验光仪的调试与操作 }\end{array}$ \\
\hline
\end{tabular}

\subsection{2. 教学单元设计}

在教学中注意光学基本知识、基本技能与医疗器械专业实 践相结合, 理论联系实际, 将知识点与能力点聚焦在理实 融合项目上。以医疗器械生命周期中的各环节作为学习情 境信息的来源, 优化设计以典型医用光学仪器为教学载体
的工作任务, 从而激发学生的学习动机。学生在教学过程 中针对医用光学仪器应用与维护实践案例中存在的问题 进行研究, 并提出解决思路。课程采用过程评价的方式调 动学生的学习积极性, 培养其独立思考能力以及发现问 题、解决问题的能力。本文以 “项目 8: 裂隙灯显微镜的 使用” 为例, 来说明教学单元设计情况, 见表 2 。

表 2 教学单元设计一一裂隙灯显微镜的使用

\begin{tabular}{|c|c|c|c|c|}
\hline 序号 & 项目 & 内容 & 学习情境信息来源 & 医疗器械生命周期 \\
\hline 1 & 教学目标 & $\begin{array}{l}\text { 1、了解裂隙灯显微镜的工作原理、光学 } \\
\text { 系统和典型的机械结构 } \\
\text { 2、学会正确调整和使用裂隙灯显微镜 }\end{array}$ & I & I \\
\hline 2 & 工作任务 & $\begin{array}{l}\text { 1、根据用户需求选择合适的裂隙灯显微 } \\
\text { 镜 } \\
\text { 、分析裂隙灯显微镜的故障, 并维修 }\end{array}$ & $\begin{array}{l}\text { 医疗器械招标采购、维 } \\
\text { 护维修、设备管理 }\end{array}$ & $\begin{array}{l}\text { 医疗器械的经营、使用、 } \\
\text { 不良事件监测、再评价、 } \\
\text { 召回 }\end{array}$ \\
\hline 3 & $\begin{array}{l}\text { 相关能力训 } \\
\text { 练 }\end{array}$ & $\begin{array}{l}\text { 1、裂隙光源部分的拆装 } \\
\text { 2、裂隙灯显微镜的安装调试 } \\
\text { 3、裂隙灯显微镜的操作 }\end{array}$ & $\begin{array}{l}\text { 医疗器械检测注册、安 } \\
\text { 装调试、临床使用 }\end{array}$ & $\begin{array}{l}\text { 医疗器械的临床试验、 } \\
\text { 注册、生产、使用 }\end{array}$ \\
\hline 4 & $\begin{array}{l}\text { 相关理论知 } \\
\text { 识 }\end{array}$ & $\begin{array}{l}\text { 1、裂隙灯显微镜原理 } \\
\text { 2、科勒照明法 } \\
\text { 3、裂隙灯显微镜检查方法 } \\
\text { 4、裂隙灯显微镜前沿技术 }\end{array}$ & $\begin{array}{l}\text { 医疗器械研究开发、市 } \\
\text { 场营销、监督管理 }\end{array}$ & $\begin{array}{l}\text { 医疗器械的研制、分类、 } \\
\text { 临床试验、注册、生产、 } \\
\text { 经营、使用、再评价 }\end{array}$ \\
\hline
\end{tabular}

\section{4. 结论}

本文根据教学主要目的要求, 分析了教学方法选取与教学 任务的关系。结合精密医疗器械技术专业背景、医疗器械 行业特色与学生未来发展, 提出了一种基于医疗器械产品 生命周期的理实融合教学方法。并通过《医用光学仪器》 课程教学举例, 详细介绍了整体设计和单元设计的课程开 发情况。该教学方法的研究与实践探索, 适应了先进制造 业重点领域特别是医疗器械领域的高素质技术应用型人 才培养的教学需求 $[8]$ 。

\section{致谢}

本文为上海健康医学院教学建设项目: 《医用光学仪器》 理实融合课程（编号: JG(19)04-A1-01) 的阶段性成果之 一。

\section{参考文献}

[1] 金才兵, 陈敬. 好课程是设计出来的 $[\mathrm{M}]$. 北京：机械 工业出版社，2015：7-11.

[2] 蒋海洪. 医疗器械法规汇编与案例精析 $[\mathrm{M}]$. 北京: 中 国法制出版社, 2016: 4-5.

[3] Heikki Kynäslahti, Pertti Kansanen, Riitta Jyrhämä, et al. The multimode programme as a variation of research-based teacher education $[\mathrm{J}]$. Teaching \& Teacher Education, 2006, 22(2):246-256.

[4] 杨开城. 课程开发: 一种技术学的视角[M]. 北京: 北 京师范大学出版社, 2018: 107-111.

[5] 精密医疗器械技术专业介绍 [Z]. http://zs.sumhs.edu.cn/9b/75/c4919a170869/page.htm, 2019-06-17.

[6] 吕维敏. 医用光学仪器应用与维护 $[\mathrm{M}]$. 北京: 人民卫 生出版社, 2011：300-306. 
[7] 杨群祥. 基于工作过程的高职教育课程开发研究

兼论国家级精品课程《商务谈判》的设计与实践 $[\mathrm{J}]$. 中国 高教研究, 2011(12): 77-78.

[8] 中国教育科学研究院课题组. 完善先进制造业重点领 域人才培养体系研究[J]. 教育研究, 2016,37(01):4-16. 